\title{
Relation Between Different Measures of Glycemic Exposure and Microvascular and Macrovascular Complications in Patients with Type 2 Diabetes Mellitus: An Observational Cohort Study
}

\author{
Rients P. T. van Wijngaarden · Jetty A. Overbeek • Edith M. Heintjes • \\ Agata Schubert · Joris Diels · Huub Straatman · Ewout W. Steyerberg • \\ Ron M. C. Herings
}

Received: June 23, 2017 / Published online: September 18, 2017

(C) The Author(s) 2017. This article is an open access publication

\section{ABSTRACT}

Introduction: This retrospective cohort study investigated the relation between different measures of glycemic exposure and micro- and macrovascular complications among patients with type 2 diabetes.

Methods: The analysis included patients receiving oral antihyperglycemic agents between 1 January 2006 and 31 December 2014 from the General Practitioner Database from the PHARMO Database Network. All recorded HbA1c levels during follow-up were used to

Enhanced content To view enhanced content for this article go to http://www.medengine.com/Redeem/ B90CF0600F2C42E0.

Electronic supplementary material The online version of this article (doi:10.1007/s13300-017-0301-4) contains supplementary material, which is available to authorized users.

R. P. T. van Wijngaarden $(\bowtie) \cdot J$. A. Overbeek · E. M. Heintjes - H. Straatman · R. M. C. Herings PHARMO Institute for Drug Outcomes Research, Utrecht, Netherlands

e-mail: rients.van.wijngaarden@pharmo.nl

J. A. Overbeek

Department of General Practice and Elderly Care Medicine, Amsterdam Public Health Research Institute, VU University Medical Centre, Amsterdam, Netherlands

A. Schubert

Janssen-Cilag Poland, Warsaw, Poland express glycemic exposure in four ways: index HbA1c, time-dependent HbA1c, exponential moving average (EMA) and glycemic burden. Association between glycemic exposure and micro-/macrovascular complications was analyzed by estimating hazard ratios and 95\% confidence intervals using an adjusted (time-dependent) Cox proportional hazards model.

Results: The analysis included 32,725 patients (median age, 65 years; $47 \%$ female). Median follow-up was 5.4 years; median number of HbA1c measurements per patient was 18.0. From all measures, HbA1c at index showed the weakest relation between all micro-/macrovascular complications, with coronary artery disease (CAD) having the highest HR $(95 \% \mathrm{CI})$ : 1.18 (1.04-1.34) for HbA1c $\geq 64 \mathrm{mmol} / \mathrm{mol}$ (8\%). The time-dependent HbA1c model showed a significant association only for microvascular complications, with retinopathy

J. Diels

Janssen Research and Development, Beerse, Belgium

E. W. Steyerberg

Erasmus MC, Rotterdam, Netherlands

R. M. C. Herings

Department of Epidemiology \& Biostatistics, VU

University Medical Centre, Amsterdam, Netherlands 
having the highest HR (95\% CI): 1.55 (1.40-1.73) for $\mathrm{HbA} 1 \mathrm{c} \geq 64 \mathrm{mmol} / \mathrm{mol}$ (8\%). EMA-defined exposure showed similar findings, although the effect of retinopathy was more pronounced [HR (95\% CI): 1.81 (1.63-2.02) for $\mathrm{HbA} 1 \mathrm{c} \geq 64 \mathrm{mmol} / \mathrm{mol}(8 \%)]$ and was also predictive for CAD [HR (95\% CI): 1.29 (1.10-1.50) for $\mathrm{HbA} 1 \mathrm{c} \geq 64 \mathrm{mmol} / \mathrm{mol}(8 \%)]$. A statistically significant relation with glycemic burden was found for all selected micro-/macrovascular complications, with retinopathy having the highest HR (95\%): 2.60 (2.19-3.07) for glycemic burden years $>3$.

Conclusion: This study shows that greater and more prolonged exposure to hyperglycemia increases the risk of micro- and macrovascular complications.

Funding: Janssen Pharmaceutica NV.

Keywords: Glycemic burden; Macrovascular; Microvascular; Type 2 diabetes

\section{INTRODUCTION}

Type 2 diabetes mellitus (T2DM) is a complex disease with many associated long-term complications and a major impact on the daily lives of patients. High glycemia shows a positive correlation with both micro- and macrovascular complications [1-3]. Therefore, to prevent or slow these complications, good glycemic control is thought to be important [4]. In general practice, glycosylated hemoglobin (HbA1c) is often used as a measure of glycemic control. The advantage of using HbA1c as opposed to blood glucose level is that it measures the average plasma glucose level over a period of 2-3 months [5]. The HbA1c target is generally $53 \mathrm{mmol} / \mathrm{mol}$ (7\%) [6]; nevertheless, T2DM patients often do not reach the recommended glycemic target and stay uncontrolled for long periods of time [7].

When assessing the risk associated with high glycemia, HbA1c measurements are often evaluated at a single point in time, which can lead to an underestimation of the role of $\mathrm{HbA1c}$ as a risk factor $[8,9]$, as it does not take the level of glycemic control over time into account. An updated mean (UM) HbA1c is sometimes used instead of the single measure as a more appropriate approximation of glycemic exposure as it is calculated as the average of all previous measurements and is recalculated if new measurements are added [1]. Using an UM has been shown to have greater predictive power $[1,10]$; however, while an UM does reflect the level of hyperglycemia over time, irregular intervals between measurements may have a significant impact. Infrequent measurements during high glycemic levels, mixed with frequent measurements during low glycemic levels, may lead to underestimation of the glycemic exposure over time and vice versa. The UM does not distinguish between recent and historic hyperglycemic periods. To allow recent glycemic levels to have more impact, more weight can be assigned to recent measurements in the UM [i.e., exponential moving average (EMA)]. However, this might also lead to diminished impact of historic periods of hyperglycemia. By calculating an area under the curve (AUC) of glycemic exposure, the effect of all measurements is taken into account, independent of whether the measurement is recent or in the past, but taking into account the duration of hyperglycemic periods as well as the extent of the hyperglycemia. This is referred to as glycemic memory [11-13].

The objective of this study is to investigate the relation between glycemic exposure and micro- and macrovascular complications among patients with T2DM using real-world data. To further investigate the effect of changes in HbA1c on long-term outcomes, different measures of glycemic exposure are used, including single point measurements, EMA and AUC.

\section{METHODS}

\section{Setting}

For this observational cohort study, data were obtained from the PHARMO Database Network. This population-based network of Dutch electronic healthcare databases combines rich, patient-centric data from different primary and secondary healthcare settings. Data from the 
General Practitioner (GP) Database were used, which cover approximately 2.5 million patients from 2005 to 2014. The GP Database comprises data from electronic patient records registered by GPs. The health records include information on diagnoses and symptoms, laboratory test results, referrals to specialists and healthcare product/drug prescriptions. The prescription records include information on the type of product, prescription date, strength, dosage regimen, quantity and route of administration. Drug prescriptions are coded according to the WHO Anatomical Therapeutic Chemical (ATC) Classification System. Diagnoses and symptoms are coded according to the International Classification of Primary Care (ICPC), which can be mapped to ICD codes, but can also be entered as free text. The study period was defined as 1 January 2006-31 December 2014.

\section{Patient Involvement}

As this was an observational study using retrospective data, patients were not involved in the study design or conduct.

\section{Study Population}

To investigate the relation between multiple measures of glycemic exposure and macro- and microvascular complications, it was important to have regularly recorded $\mathrm{HbA1c}$ measurements. Diabetes care in The Netherlands is mainly managed in the primary care setting and guided by the Dutch GP guideline for T2DM [14]. It is well organized, including regular checkups. For our study, only general practices adhering to these regular checkups were selected, which was defined as having at least $50 \%$ of its T2DM patients with at least three HbA1c measurements per year. This selection was done at the practice level instead of patient level to avoid biased selection as well-managed patients with T2DM may be monitored less frequently.

Within the selected practices, T2DM patients were identified as having either a diagnosis code (ICPC code T90.02) or at least two consecutive prescriptions of oral antihyperglycemic agents (AHA; ATC code A10B) within a 6-month period at any time in the available medication records. The index date was defined as the first oral AHA prescription after at least 1 year of enrollment. Patients without any oral AHA prescriptions during the study period were excluded as well as patients with diagnoses for T1DM, gestational diabetes or polycystic ovary syndrome (PCOS). Only patients between 40 and 84 years of age at the index date were included. The most recent HbA1c measurement within 1 year prior to or at the index date was defined as the index HbA1c measurement. If an index $\mathrm{HbA} 1 \mathrm{c}$ measurement was not available, the patient was excluded. Further exclusions were based on the event of interest. Patients with a prior event were excluded for the analysis of that specific event but were possibly included in other analyses. Finally, patients were required to have at least one HbA1c measurement during follow-up. Again, this could differ per event of interest, since the end of follow-up was determined as either the first occurrence of an event, death, loss to follow-up in the PHARMO Database Network or end of the study period, whichever occurred first.

The following characteristics were determined: age at index date, gender, HbA1c at index date, the proportion of prior complications at index date, newly treated, follow-up, number of HbA1c measurements during follow-up and BMI at the index date. Newly treated patients were defined as those without any AHA prescription in the year prior to the index date.

\section{Outcomes}

Diabetic foot, retinopathy and renal complications were the microvascular events of interest, and coronary artery disease (CAD) and cerebrovascular disease were the macrovascular events of interest. All complications were based on GP-recorded information.

\section{Microvascular Complications}

Diabetic foot was identified based on recorded codings and text searches for the diagnoses of necrosis, ulcer, diabetic foot, infection, wound, erysipelas, gangrene, Charcot foot, loss of 
protective sensation, peripheral artery disease, peripheral arterial occlusive disease, claudication, neuropathy and amyotrophy $[15,16]$. The positive clinical assessments of necrosis and ulcers were also included.

Retinopathy was identified based on ICPC code F83 (retinopathy), positive outcomes of retinopathy assessments and text searches for the diagnoses of retinopathy, maculopathy or diabetic macular edema.

Renal complications were identified based on multiple assessments of the estimated glomerular filtration rate $<60 \mathrm{ml} / \mathrm{min} / 1.73 \mathrm{~m}^{2}$ [modification of the Diet in Renal Disease (MDRD) formula] or proteinuria (albumin $>30 \mathrm{mg} / \mathrm{l}$ ), at least 90 days and at most 365 days apart, or on searches for the diagnoses of nephropathy, nephrotic syndrome, glomerular hematuria, glomerulonephritis, nephrosis, kidney failure, dialysis, transplants, proteinuria and albuminuria.

\section{Macrovascular Complications}

The identification of CAD was based on ICPC codes K53 (coronary artery angioplasty), K74 (angina pectoris), K75 (myocardial infarction) and K76 (ischemic heart disease) and searches for the diagnoses of angina pectoris, acute coronary syndrome (ACS) and myocardial infarction. Cerebrovascular disease-related events were identified based on ICPC codes K89 [transient ischemic attack (TIA)] and K90 [stroke/cerebrovascular accident (CVA)] as well as searches for the diagnoses of stroke, cerebral infarction, CVA and TIA.

\section{Glycemic Exposure}

Four different definitions of glycemic exposure were studied. A first definition of exposure was based on the HbA1c at index $[3,17,18]$, defined as the last HbA1c measurement in the year prior to the index date. A second definition of glycemic exposure was based on all observed HbA1c measures during post-index follow-up; HbA1c was included as a time-dependent covariate. The third approach was similar to the previous one, but instead of including observed HbA1c values, the exponential moving average (EMA) of HbA1c was included as a time-dependent covariate. The exponential moving average (EMA) is a variant of the updated mean (UM) $[1,17]$. In this measure, the current HbA1c measurement amounts to $20 \%$ of the calculated value, and the previously calculated EMA amounts to 80\%. The EMA was chosen to ensure that, independent of the total number of measurements, the last measurement is given a fixed weight of 0.2. In a fourth approach, the glycemic burden was calculated, defined as the AUC given a threshold of $53 \mathrm{mmol} / \mathrm{mol}$ (7\%) [13]. Between two HbA1c measurements a linear interpolation was used, and the glycemic burden over a time period was calculated when either a new HbA1c measurement occurred or the age of a patient changed. At the start of a given time period, the cumulative glycemic burden was defined as the sum of all the calculated AUCs up until then. Furthermore, the cumulative glycemic burden is divided by 365 to express the value in years. The calculation of the glycemic burden years (GBY) is presented graphically in Fig. 1. The GBY was included as a time-dependent variable in the Cox-model.

Partial GBYs ( $\mathrm{AUC}_{1}, \mathrm{AUC}_{2}$, etc.) were calculated per interval between cutoff points as the difference in HbA1c above the threshold of $53 \mathrm{mmol} / \mathrm{mol}(7 \%)$ multiplied by the number of years between those points. Cutoff points were HbA1c measurements, changes in age or interpolation lines crossing the threshold. Cumulative GBY was updated at each of the cutoff points by summing all prior GBYs.

\section{Statistical Analysis}

Population characteristics were reported as absolute and relative frequencies $(n, \%)$ for categorical variables and as median and interquartile range (IQR) for continuous variables, if applicable. Incidence rates (IRs) were calculated per type of event by dividing the number of patients with an event by the total number of patient-years at risk (summed durations of total treatment period, censored at the time of the event under investigation).

A Cox proportional hazards regression analysis was performed for each of the micro- and 


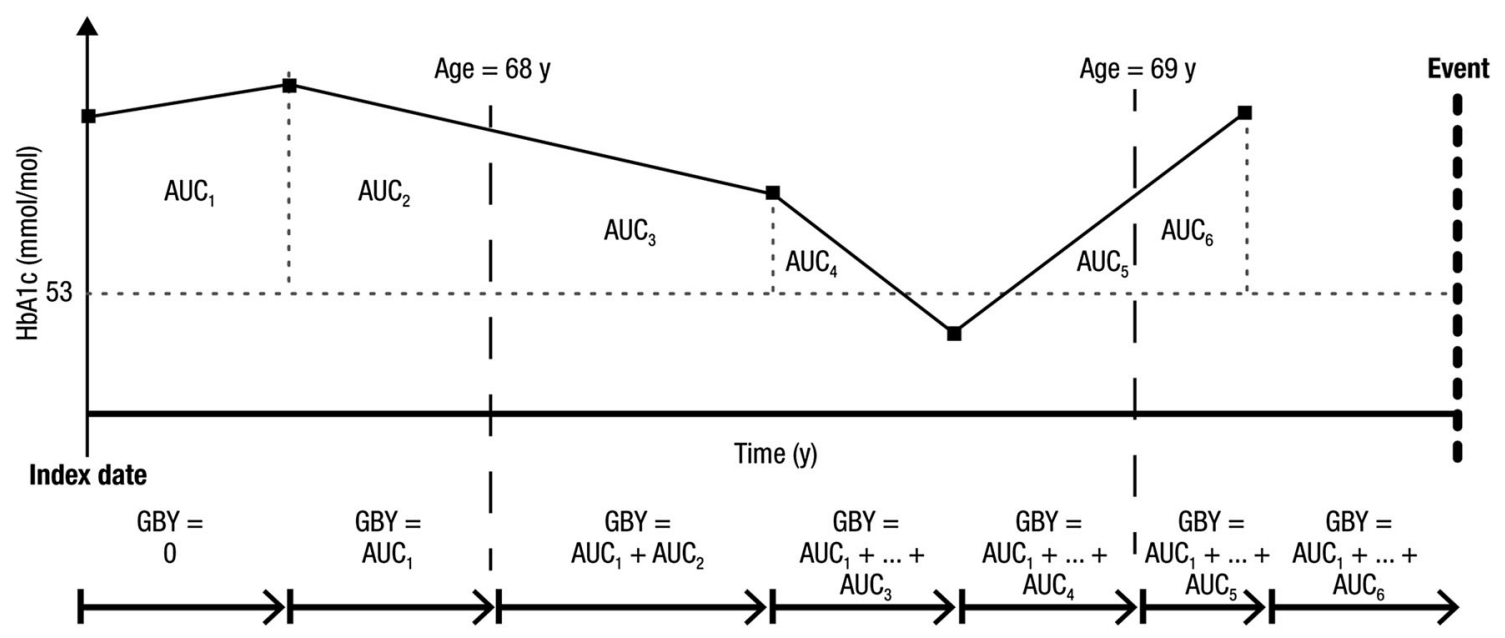

Fig. 1 Calculation of GBYs. $A U C$ area under the curve, $G B Y$ glycemic burden year

macrovascular complications with each of the four glycemic exposure measures. The PROC PHREG procedure from SAS 9.4 was used.

For the model, using HbA1c at index, the Cox proportional hazards regression model was adjusted for age, gender and the year of index. For illustration, the $\mathrm{HbA1c}$ value at index was categorized as $<53 \mathrm{mmol} / \mathrm{mol}(7 \%), 53$ to $<64 \mathrm{mmol} / \mathrm{mol} \quad(7 \%$ to $<8 \%)$ and $\geq 64 \mathrm{mmol} / \mathrm{mol}$ (8\%). For the other models, repeated measurements of HbA1c were included, and a time-dependent Cox proportional hazards regression analysis was performed. Age and the measure of exposure (HbA1c, EMA or GBY) were used as time-dependent variables, and the model was corrected for age, gender, the year of index and time (in years) since the first measurement above $53 \mathrm{mmol} / \mathrm{mol} \mathrm{(7 \% )}$ and the time (in years) since the last measurement below $53 \mathrm{mmol} / \mathrm{mol}$ (7\%). The timedependent HbA1c and the EMA were categorized as follows: $<53 \mathrm{mmol} / \mathrm{mol}(7 \%), 53$ to $<64 \mathrm{mmol} / \mathrm{mol} \quad(7 \%$ to $<8 \%)$ and $\geq 64 \mathrm{mmol} / \mathrm{mol}$ (8\%). The categorization of glycemic burden was based on its distribution: 0 (reference), $>0$ to $\leq 1,>1$ to $\leq 3$ and $>3$ GBYs.

This article does not contain any new studies with human or animal subjects performed by any of the authors. No ethics committee approval or informed consent was required for this anonymized database study.

\section{RESULTS}

\section{Patients}

The flow chart describing the process of patient selection is shown in Fig. 2. After applying all inclusion and exclusion criteria, approximately 30,000 patients were included per outcome. The characteristics of the total population, without exclusions of patients with prior events, are shown in Table 1. Median (IQR) age was 65 (57-73) years, and approximately half of the patients were female (47\%). At index date, $50 \%$ of the patients were on target $[\mathrm{HbA} 1 \mathrm{c}<53$ $\mathrm{mmol} / \mathrm{mol}(7 \%)]$, and only $18 \%$ of the patients had an HbA1c at index $\geq 64 \mathrm{mmol} / \mathrm{mol}(8 \%)$.

\section{Glycemic Exposure}

In total, 20 analyses were performed, and the results are presented in Fig. 3 and summarized in Supplemental Table S1. The results of the different events are plotted separately, with the hazard ratios (HRs) on the vertical axis. Within each plot, the four different measures of glycemic exposure are displayed with the HR per categorized variable.

The results for the first model, using only the HbA1c at index, suggested an increased risk for higher HbA1c values for the microvascular 


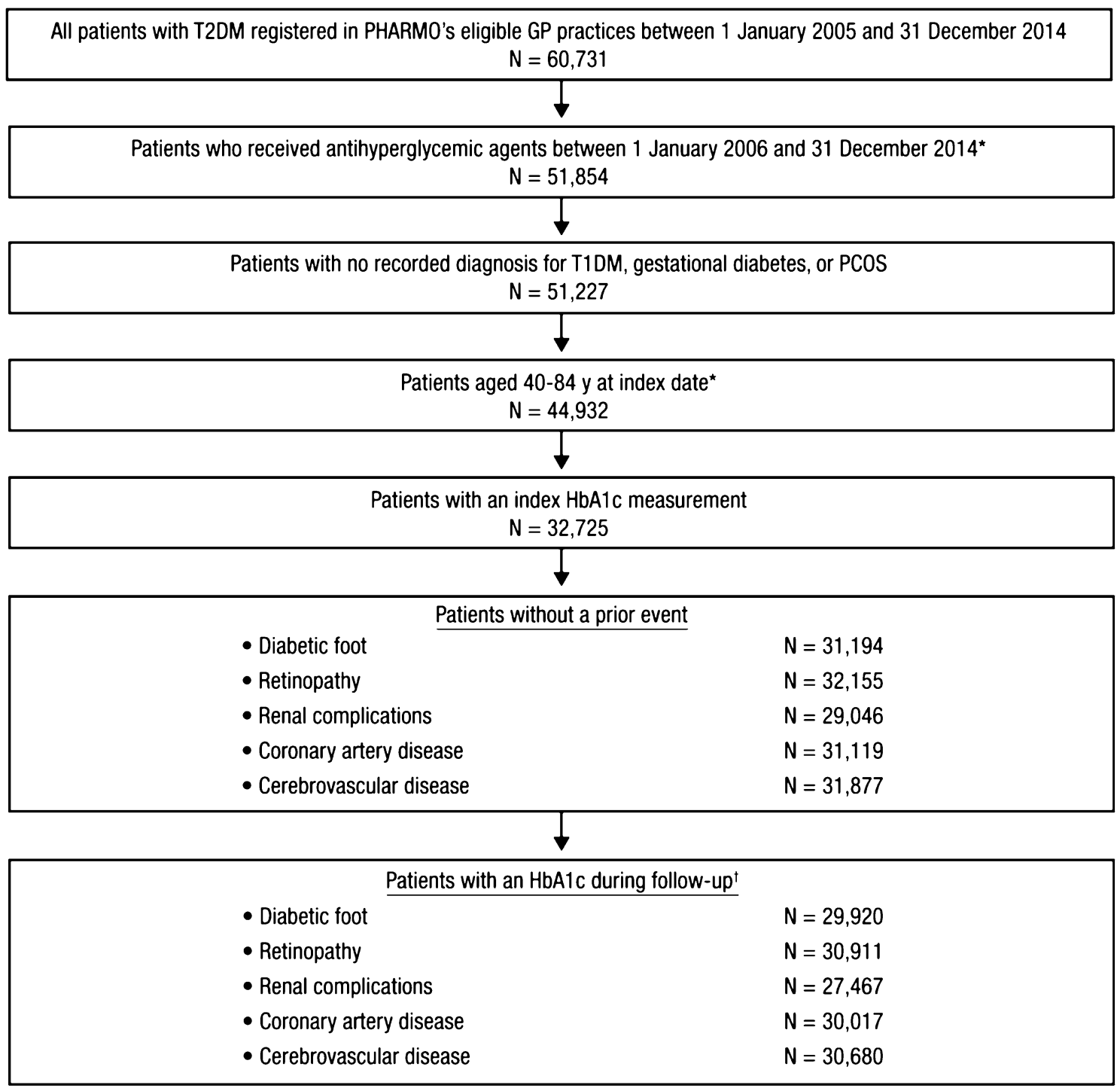

Fig. 2 Flow chart of patient selection. GP general practitioner, PCOS polycystic ovary syndrome, T1DM type 1 diabetes mellitus, T2DM type 2 diabetes mellitus. ${ }^{*}$ The first antihyperglycemic prescription after at least 1 year of enrollment in the database. ${ }^{\dagger}$ Follow-up differed per event

complications as well as CAD. However, the results were only significant for the highest category $[\mathrm{HbA} 1 \mathrm{c} \geq 64 \mathrm{mmol} / \mathrm{mol}(8 \%)]$ for renal complications [HR (95\% CI) of 1.10 (1.02-1.18)] and CAD [HR (95\% CI) of $1.18(1.04-1.34)]$ compared to the reference category [HbA1c $<53 \mathrm{mmol} / \mathrm{mol}(7 \%)]$.

The results for the time-dependent $\mathrm{HbA1c}$ model showed an increased risk for both of interest as patients were followed from the index date to end of follow-up [i.e., first occurrence of the event of interest, death, loss to follow-up in the PHARMO Database Network or end of the study period (31 December 2014), whichever occurred first]

diabetic foot and retinopathy. For diabetic foot, the HRs $(95 \% \mathrm{CI})$ for the middle and highest HbA1c categories were 1.09 (1.00-1.19) and 1.36 (1.21-1.52), respectively. For retinopathy, this was 1.19 (1.10-1.30) and 1.55 (1.40-1.73), respectively. For renal complications, only the highest HbA1c category showed a significant association [HR (95\% CI): 1.20 (1.10-1.31)]. The associations between time-dependent HbA1c 
Table 1 Characteristics of patients with T2DM

\begin{tabular}{|c|c|}
\hline & $\begin{array}{l}\text { Total } \\
N=32,725\end{array}$ \\
\hline \multicolumn{2}{|l|}{ Age at index date, years } \\
\hline Median (IQR) & $65(57-73)$ \\
\hline \multicolumn{2}{|l|}{ Gender, $n(\%)$} \\
\hline Male & $17,270(53)$ \\
\hline Female & $15,455(47)$ \\
\hline \multicolumn{2}{|l|}{ HbAlc at index date, $n(\%)$} \\
\hline$<53 \mathrm{mmol} / \mathrm{mol}(7 \%)$ & $16,325(50)$ \\
\hline $\begin{array}{l}53 \mathrm{mmol} / \mathrm{mol}(7 \%) \text { to }<58 \mathrm{mmol} / \mathrm{mol} \\
(7.5 \%)\end{array}$ & $6582(20)$ \\
\hline $\begin{array}{l}58 \mathrm{mmol} / \mathrm{mol}(7.5 \%) \text { to }<64 \mathrm{mmol} / \mathrm{mol} \\
(8 \%)\end{array}$ & $3771(12)$ \\
\hline$\geq 64 \mathrm{mmol} / \mathrm{mol}(8 \%)$ & $6047(18)$ \\
\hline Median (IQR) & $\begin{array}{l}53.0 \\
\quad(46.4-59.6)\end{array}$ \\
\hline \multicolumn{2}{|l|}{ Prior complications at index date, $n(\%)$} \\
\hline \multicolumn{2}{|l|}{ Microvascular } \\
\hline Diabetic foot & $1531(5)$ \\
\hline Retinopathy & $570(2)$ \\
\hline Renal complications & $3679(11)$ \\
\hline \multicolumn{2}{|l|}{ Macrovascular } \\
\hline Coronary artery disease & $1526(5)$ \\
\hline Cerebrovascular disease & $848(22)$ \\
\hline \multicolumn{2}{|l|}{ Newly treated } \\
\hline Yes & $13,656(42)$ \\
\hline No & $19,069(58)$ \\
\hline \multicolumn{2}{|l|}{ Follow-up, years } \\
\hline Median (IQR) & $5.4(2.5-7.8)$ \\
\hline \multicolumn{2}{|l|}{ Number of HbAlc measurements } \\
\hline Median (IQR) & $\begin{array}{l}18.0 \\
\quad(8.0-29.0)\end{array}$ \\
\hline BMI, $\mathrm{kg} / \mathrm{m}^{2 \mathrm{a}}$ & \\
\hline
\end{tabular}

Table 1 continued

\begin{tabular}{lc}
\hline & $\begin{array}{l}\text { Total } \\
\boldsymbol{N}=\mathbf{3 2 , 7 2 5}\end{array}$ \\
\hline Median (IQR) & 29.7 \\
& $(26.8-33.3)$ \\
\hline
\end{tabular}

$B M I$ body mass index, $I Q R$ interquartile range, $T 2 D M$ type 2 diabetes mellitus

a BMI was determined in the year prior to the index date and was available for $65 \%$ of the patients. The incidence rates of the micro- and macrovascular events are presented in Table 2. The incidence rate of microvascular events ranges from 2.5 new retinopathy cases per 100 patient-years (PYs) to 6.0 new renal complication cases per 100 PYs, and the incidence rates of macrovascular events were between 1 and 1.4 events per 100 PYs

and macrovascular complications were not significant.

The results for the third model, using the EMA, were very similar to the results of the previous model with a few notable exceptions. Specifically, the HRs for retinopathy were markedly higher for both the middle and highest HbA1c categories [i.e., HRs $(95 \% \mathrm{CI})$ of $1.29(1.18-1.41)$ and 1.81 (1.63-2.02), respectively]. Also, the results for CAD were significant for the highest category $[\mathrm{HbA} 1 \mathrm{c} \geq 64 \mathrm{mmol} / \mathrm{mol}$ (8\%); HR (95\% CI) of $1.29(1.10-1.50)]$.

The results for the glycemic burden model showed significantly increased risks associated with higher GBY levels for the microvascular complications. For diabetic foot, the HRs were $1.30,1.46$ and 2.15 for $>0$ to $\leq 1,>1$ to $\leq 3$ and $>3$ GBYs, respectively, compared to the reference category of no glycemic burden. For retinopathy, the HRs ranged from 1.41 to 2.60, and for renal complications, the HRs ranged from 1.20 to 1.61 . The macrovascular complications showed similar results. However, for coronary artery disease GBY $>1$ to $\leq 3$, the HR was not significantly higher. Cerebrovascular disease on the other hand did show a dose response relation where the HRs ranged from 1.20 to 1.52 .

Additional results of the models, including the covariates, are included in Online Resource 1 (Supplemental Table S2). 

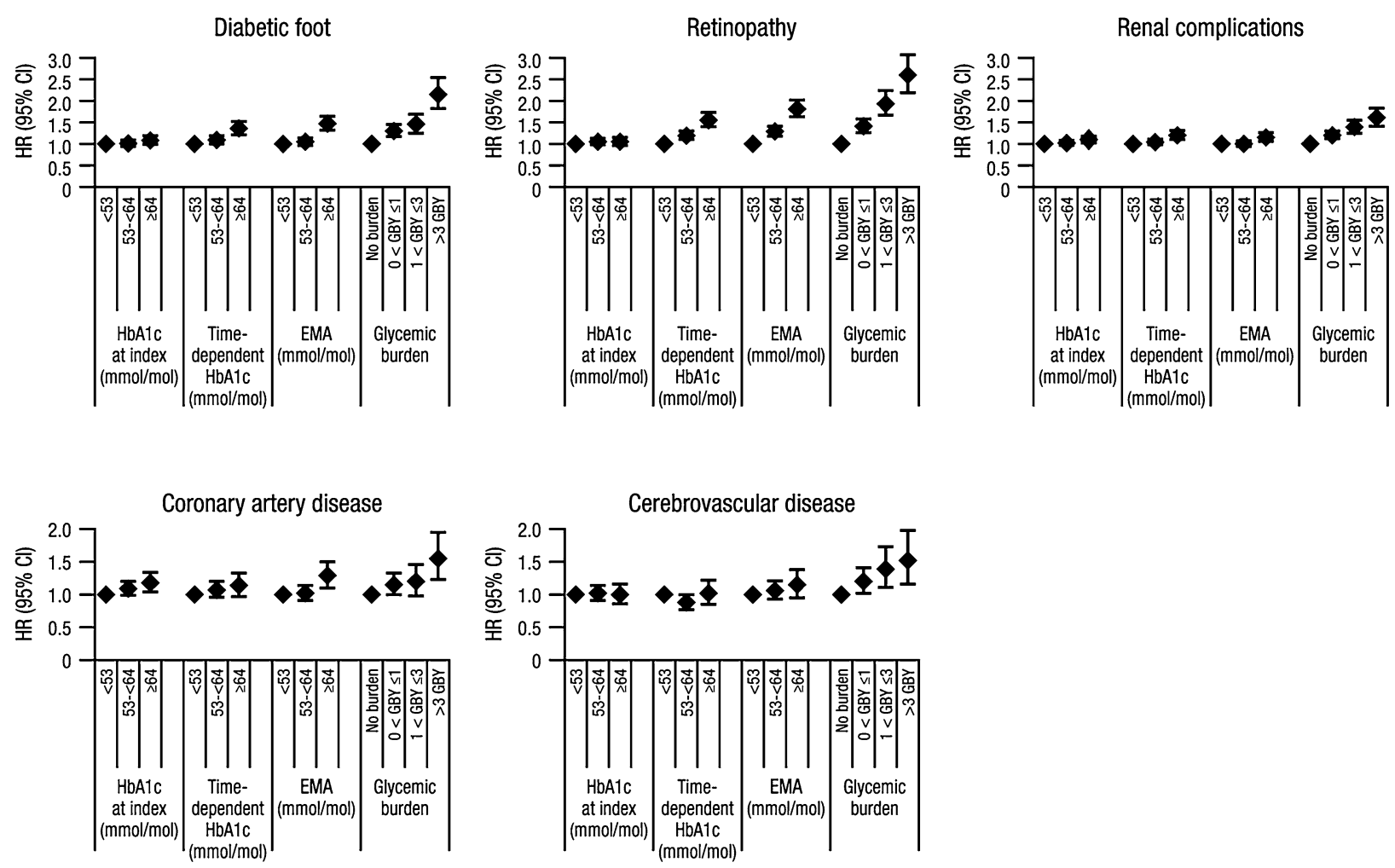

Fig. 3 Association between different measures for glycemic exposure and micro- and macrovascular complications. $C I$ confidence interval, EMA exponential moving average, $H R$ hazard ratio

Table 2 Incidence rates $(95 \% \mathrm{CI})$ of micro- and macrovascular complications

\begin{tabular}{lllll}
\hline & $N_{\text {at risk }}$ & PY $_{\text {at risk }}$ & $\boldsymbol{N}_{\text {event }}$ & IR/100 PY (95\% CI) \\
\hline Microvascular complications & & & & $2.65(2.57-2.73)$ \\
Diabetic foot & 31,194 & 147,440 & 3904 & $2.50(2.42-2.58)$ \\
Retinopathy & 32,155 & 150,465 & 3764 & $6.02(5.88-6.16)$ \\
Renal complications & 29,046 & 124,708 & 7507 & $1.40(1.34-1.46)$ \\
Macrovascular complications & & & & $1.06(1.01-1.11)$ \\
Coronary artery disease & 31,199 & 151,921 & 2129 & 1664 \\
Cerebrovascular disease & 31,877 & 156,966 & & \\
\hline
\end{tabular}

$C I$ confidence interval, $I R$ incidence rate, $P Y$ patient-year

\section{DISCUSSION}

\section{Main Findings}

All micro- and macrovascular complications were weakly associated with exposure to high
$\mathrm{HbA} 1 \mathrm{c}[\mathrm{HbA} 1 \mathrm{c} \geq 64 \mathrm{mmol} / \mathrm{mol}(8 \%)]$ at index to some extent, but this was only significant for renal complications [HR $(95 \%$ CI $)$ of 1.10 (1.02-1.18)] and CAD [HR (95\% CI) of 1.18 (1.04-1.34)]. When modeling glycemic exposure as the time-dependent observed $\mathrm{HbA1c}$, a significantly increased risk for all microvascular 
complications was observed for HbA1c $\geq 64 \mathrm{mmol} / \mathrm{mol}$ (8\%). For the macrovascular events, this model was inconclusive, and the results were not significant. These results are largely in line with other findings. For renal complications, Gerstein et al. [17] found an association between an increase in $\mathrm{HbA1c}$ at index and nephropathy, and Klein [3] found an association between an increase in $\mathrm{HbA1c}$ at index and both gross proteinuria and renal failure. For diabetic foot, an increased risk was expected for the model using the HbA1c value at index in concordance with earlier findings $[3,18]$; however, these studies found associations with either amputation [3] or sensory neuropathy [18], while we defined a composite endpoint covering a broad spectrum of peripheral arterial problems. However, our findings do suggest that the most recent $\mathrm{HbA1c}$ measurement (i.e., time-dependent HbA1c) is a better predictor for diabetic foot than the HbA1c at index. For CAD, the results regarding HbA1c at index are in concordance with the literature [10] where an HR (95\% CI) of 1.05 (1.00-1.10) was reported for a $1 \%$ increase in HbA1c also using the HbA1c at index. On the other hand, no significant effect was found for the time-dependent HbA1c, suggesting that the $\mathrm{HbA1c}$ at index is a better predictor for CAD than the most recent HbA1c measurement. The results for cerebrovascular disease were inconclusive, which is in line with other findings [10] where an HR (95\% CI) of $0.96(0.88-1.06)$ for a $1 \%$ increase of $\mathrm{HbA} 1 \mathrm{c}$ at index was reported. The EMA-based results for the microvascular events showed similar results as the time-dependent HbA1c. Retinopathy specifically showed a clear dose response for EMA, and associations were stronger than in the time-dependent HbA1c model. For the macrovascular event of coronary artery disease, a significantly increased risk was observed in the highest risk category $[\mathrm{EMA} \geq 64 \mathrm{mmol} / \mathrm{mol}(8 \%)]$. Again, the results for cerebrovascular disease were inconclusive. An association between the increase in updated mean $\mathrm{HbA} 1 \mathrm{c}$ and the risk has been reported for diabetic foot [1], retinopathy $[8,19]$, renal failure [17], CAD $[1,2]$ and cerebrovascular disease [1]. With the exception of cerebrovascular disease, our results reflected previously reported findings. Absence of an association between EMA and the risk of cerebrovascular disease may be explained by the fact that we defined cerebrovascular disease as a composite endpoint including stroke, TIA, CVA and cerebral infarction, while Stratton et al. [1] reported an HR (95\% CI) of 1.14 (1.01-1.27) for a $1 \%$ increase in updated mean HbA1c for stroke only. For the microvascular events, the analyses using glycemic burden showed the clearest dose response. Overall, the HRs were higher, but the categories used were different from the previous models and not directly comparable as the GBY categories of this cumulative outcome were based on the distribution of the number of patients rather than clinically recognizable cutoff points for HbA1c. Again, the effects for retinopathy were stronger than for the other microvascular events: the HRs ranged from 1.41 to 2.60 against $1.30-2.15$ for diabetic foot and 1.20-1.61 for renal failure. Maple-Brown et al. [13] used the AUC as a measure for chronic glycemic exposure and found that it was a good predictor for microvascular complications. For the macrovascular events, CAD (GBY $>0$ to $\leq 1$ and GBY $>3$ ) showed a significant association. Cerebrovascular disease showed a significant, albeit small, dose response with HRs ranging from 1.20 to 1.51 .

\section{Strengths and Limitations}

A strength of the study is the use of a large population in a real-world setting with long follow-up. Data from GPs in The Netherlands are especially suited, since diabetes care in The Netherlands is highly organized, and the majority (90\%-95\%) of T2DM patients are treated within the primary care setting. This means that we were able to validate our models in actual clinical practice.

A limitation is that the duration of diabetes was not included in the model because it is not always explicitly recorded as a clinical parameter. We also did not differentiate between patients who were and were not newly treated. Both have resulted in underestimation of the glycemic burden and are likely to have resulted in an underestimation of the associations. 
However, available data reflect the situation in the daily practice of any physician, who will not always know the glycemic history of a patient and will act on the data available at the time of a visit. Furthermore, we performed post hoc analyses, correcting for newly initiated or prevalent use of AHA at the time of the index date, to test whether underestimation of the glycemic burden of patients that were already treated at the start of their recorded history might have caused underestimation of the associations with complications. The results were very similar to the main analysis and therefore could not support this assumption. This means that even with incomplete records, all available history of a patient may provide valuable information to a physician regarding the build-up of glycemic memory. One of the limitations of real-world evidence in general is that information is not collected or recorded for the purpose of doing research. Changes in disease management have had a major impact on the way complications were recorded during the study period, leading to more detailed outcome recording over time. To allow analysis of more or less similar outcomes over time, composite endpoints had to be used. For example, in the case of diabetic foot, this resulted in a mix of micro- and macrovascular causes. This loss of specificity may have weakened the association of specific contributing factors within the composite. Future studies may be able to clarify specific associations by using more specific outcome definitions. In 2006, an integrated diabetes care program was introduced, with structured management and regular checkups of the patient by an interdisciplinary team of GPs, diabetes nurses, podiatrists and ophthalmologists. Check-ups include assessment of HbA1c, renal function, retinopathy, (early) signs of diabetic foot and history taking with regard to macrovascular complications. The gradual rollout of this integrated care was boosted by financial incentives linked to developing performance indicators offered by healthcare insurance companies to GPs from mid-2007 onward. This is reflected in the data by a steady increase in the recorded number of complications, which was about fivefold over the whole study period. As $40 \%$ of our population had an index date before 2008 , this means that a significant number of patients may have been misclassified as having no complications at the index date and that some complications now registered as newly developed may have been pre-existing. Incidence rates may therefore have been overestimated. As this possible misclassification was most likely irrespective of HbA1c levels, we do not believe that this non-differential misclassification has influenced our results to a large extent. Finally, we have not taken treatment into account, while the type of treatment may influence outcomes through other mechanisms than glucose lowering. For future research, it would be very valuable to take the type of treatment and other potential risk factors into account.

\section{Implications for Clinical Practice}

The first two models used a single point measurement as a predictor for outcomes, where this single point can either be static (i.e., HbA1c at index) or time updating (i.e., time-dependent HbA1c). The static model is highly practical for database studies, while the time-updating model better reflects the interpretation a physician might apply in daily practice (i.e., use of the last known value for risk assessment). However, it should be noted that the results of the current study show that neither of these models is necessarily a good representation of the risk to which an individual is exposed. The EMA, on the other hand, shows a stronger relation with diabetic foot, retinopathy and $\mathrm{CAD}$, if not with the other outcomes, which does suggest that glycemic memory plays a role in these outcomes. This is further corroborated by the results of the glycemic burden model where these associations are more pronounced and even become apparent for the macrovascular complications. Given that the glycemic burden model gives a better discrimination between patients that do or do not develop complications, it is a very promising method for further research into the long term effects of hyperglycemia. This could contribute valuable evidence to influence clinical decision making. All in all, the results from this study show that 
longer exposure to HbA1c levels above target is associated with increased risk of both microand macrovascular complications. Furthermore, the results suggest that glycemic memory plays a role in the development of diabetic complications and that treatment inertia increases the risk of these outcomes in broad populations of patients with T2DM who are not on target [20]. From the individual patient perspective, however, it is important to note that intensive glucose-lowering treatment aiming at a low target HbA1c may lead to increased risk of hypoglycemia or adverse drug reactions and therefore is not recommended for patients of older age or with short life expectancy, important comorbidities or poor self-care capabilities [6]. This is why the most recent revision of the diabetes guidelines for T2DM [21] has included higher targets for patients over 70 years of age who cannot be managed with metformin alone. Our finding that glycemic memory plays an important role in the development of complications emphasizes the importance of physicians keeping in mind that lower targets are probably more beneficial for a patient, provided that they are attainable without the trade-off of increased immediate risk. This underlines the need for frequent and active monitoring of the diabetes patient.

\section{CONCLUSION}

This study shows that greater and more prolonged exposure to hyperglycemia is associated with increased risk of both micro- and macrovascular complications. The possibility of damage caused by clinical inertia is something that needs to be addressed in clinical practice.

\section{ACKNOWLEDGEMENTS}

The authors would like to thank all the healthcare providers contributing information to the database included in this study. This work was supported by the Dutch Diabetes Research Foundation (grant no. 2014.00.1753) and sponsored by Janssen Pharmaceutica NV. Sponsorship for this study and article processing charges were funded by Janssen Pharmaceutica NV. Technical editorial assistance was provided by Shannon O'Sullivan, ELS, of MedErgy and was funded by Janssen Pharmaceutica NV. All named authors meet the International Committee of Medical Journal Editors (ICMJE) criteria for authorship for this manuscript, take responsibility for the integrity of the work as a whole and have given final approval to the version to be published. Rients P. T. van Wijngaarden, Jetty A. Overbeek, Edith M. Heintjes, Agata Schubert, Joris Diels, Huub Straatman, Ewout W. Steyerberg and Ron M. C. Herings contributed to the conception and the design of this study. Rients P. T. van Wijngaarden, Jetty A. Overbeek and Huub Straatman performed the analyses. Rients P. T. van Wijngaarden is the guarantor of the study. Rients $P$. $T$. van Wijngaarden (the manuscript's guarantor) affirms that the manuscript is an honest, accurate and transparent account of the study being reported, that no important aspects of the study have been omitted and that any discrepancies from the study as planned (and, if relevant, registered) have been explained.

Disclosures. The PHARMO Institute for Drug Outcomes Research reports grants from Janssen-Cilag. Rients P.T van Wijngaarden is an employee of the PHARMO Institute. Jetty A. Overbeek is an employee of the PHARMO Institute. Edith M. Heintjes is an employee of the PHARMO Institute. Huub Straatman is an employee of the PHARMO Institute. Ron M.C. Herings $\mathrm{H}$. is an employee of the PHARMO Institute. The PHARMO Institute is an independent research institute and performs financially supported studies for government and related healthcare authorities and several pharmaceutical companies. Agata Schubert is a full-time employee of Janssen-Cilag Poland. Joris Diels is a full-time employee of Janssen Research \& Development. Ewout W. Steyerberg has nothing to disclose.

Compliance with Ethics Guidelines. This article does not contain any new studies with human or animal subjects performed by any of the authors. 
Data Availability. Requests for sharing study data must be made on specific grounds either with the aim to corroborate the study results in the interest of Public Health or in the context of an audit by a competent authority. Sufficient information needs to be provided to confirm that the request is made for one of the above-mentioned purposes, including a sound justification and, in case of a request with a view to corroborate study results, a protocol on the research for which the data will be used or a plan for quality control checks, as applicable.

Open Access. This article is distributed under the terms of the Creative Commons Attribution-NonCommercial 4.0 International License (http://creativecommons.org/licenses/ by-nc/4.0/), which permits any noncommercial use, distribution, and reproduction in any medium, provided you give appropriate credit to the original author(s) and the source, provide a link to the Creative Commons license, and indicate if changes were made.

\section{REFERENCES}

1. Stratton IM, Adler AI, Neil HAW, Matthews DR, Manley SE, Cull CA, et al. Association of glycaemia with macrovascular and microvascular complications of type 2 diabetes (UKPDS 35): prospective observational study. BMJ. 2000;321:405-12.

2. Zhang Y, Hu G, Yuan Z, Chen L. Glycosylated hemoglobin in relationship to cardiovascular outcomes and death in patients with type 2 diabetes: a systematic review and meta-analysis. PLoS One. 2012; 7:e42551.

3. Klein R. Hyperglycemia and microvascular and macrovascular disease in diabetes. Diabetes Care. 1995;18:258-68.

4. UK Prospective Diabetes Study (UKPDS) Group. Intensive blood-glucose control with sulphonylureas or insulin compared with conventional treatment and risk of complications in patients with type 2 diabetes (UKPDS 33). Lancet. 1998;352:837-53.

5. Jeffcoate SL. Diabetes control and complications: the role of glycated haemoglobin, 25 years on. Diabet Med. 2004;21:657-65.
6. Inzucchi SE, Bergenstal RM, Buse JB, et al. Management of hyperglycemia in type 2 diabetes, 2015: a patient-centered approach: update to a position statement of the American Diabetes Association and the European Association for the Study of Diabetes. Diabetes Care. 2015;38:140-9.

7. Khunti K, Wolden ML, Thorsted BL, Andersen M, Davies MJ. Clinical inertia in people with type 2 diabetes: a retrospective cohort study of more than 80,000 people. Diabetes Care. 2013;36:3411-7.

8. Yoshida Y, Hagura R, Hara Y, Sugasawa G, Akanuma Y. Risk factors for the development of diabetic retinopathy in Japanese type 2 diabetic patients. Diabetes Res Clin Pract. 2001;51:195-203.

9. Araki S, Haneda M, Sugimoto T, Isono M, Isshiki K, Kashiwagi A, et al. Factors associated with frequent remission of microalbuminuria in patients with type 2 diabetes. Diabetes. 2005;54:2983-7.

10. Lind M, Odén A, Fahlén M, Eliasson B. A systematic review of HbA1c variables used in the study of diabetic complications. Diabetes Metab Syndr. 2008;2:282-93.

11. Bailey CJ, Day C. Glycaemic memory. Br J Diabetes Vasc Dis. 2008;8:242-7.

12. Lind $M$, Oden A, Fahlen $M$, Eliasson B. The true value of HbA1c as a predictor of diabetic complications: simulations of HbA1c variables. PLoS One. 2009;4:e4412.

13. Maple-Brown LJ, Ye C, Retnakaran R. Area-under-the-HbA1c-curve above the normal range and the prediction of microvascular outcomes: an analysis of data from the Diabetes Control and Complications Trial. Diabet Med. 2013;30:95-9.

14. Rutten GEHM, De Grauw WJC, Nijpels G, et al. NHG-Standaard Diabetes mellitus type 2 (Tweede herziening). Huisarts Wet. 2006;49:137-52.

15. Jain AKC, Joshi S. Diabetic foot classifications: review of literature. Med Sci. 2013;2:715-21.

16. Fincke BG, Miller DR, Turpin R. A classification of diabetic foot infections using ICD-9-CM codes: application to a large computerized medical database. BMC Health Serv Res. 2010;10:192.

17. Gerstein HC, Pogue J, Mann JF, et al. The relationship between dysglycaemia and cardiovascular and renal risk in diabetic and non-diabetic participants in the HOPE study: a prospective epidemiological analysis. Diabetologia. 2005;48:1749-55.

18. Adler AI, Boyko EJ, Ahroni JH, Stensel V, Forsberg RC, Smith DG. Risk factors for diabetic peripheral sensory neuropathy. Results of the Seattle 
Prospective Diabetic Foot Study. Diabetes Care. 1997;20:1162-7.

19. Araki A, Ito H, Hattori A, Inoue J, Sato T, Shiraki M, et al. Risk factors for development of retinopathy in elderly Japanese patients with diabetes mellitus. Diabetes Care. 1993;16:1184-6.

20. Cardoso CR, Leite NC, Ferreira MT, Salles GF. Prognostic importance of baseline and serial glycated hemoglobin levels in high-risk patients with type 2 diabetes: the Rio de Janeiro Type 2 Diabetes Cohort Study. Acta Diabetol. 2015;52:21-9.

21. Rutten GEHM, de Grauw W, Nijpels G, et al. NHG-Standaard Diabetes mellitus type 2 (derde herziening). Huisarts Wet. 2013;56:512-25. 